Gena R. Ghearing

Thomas M. Munger

Allan S. Jaffe

Eduardo E. Benarroch

Jeffrey W. Britton

\title{
Clinical cues for detecting ictal asystole
}

Abstract Objective The occurrence of asystole during an epileptic seizure is the most dramatic manifestation of ictal bradycardia. Recognition of ictal asystole is important as treatment with both antiepileptic drugs and cardiac pacing may be necessary. The purpose of this study was to identify clinical cues to aid in the detection of ictal asystole. Methods We analyzed the clinical and electrophysiologic features of all recorded seizures in consecutive patients diagnosed with ictal bradycardia and asystole on prolonged videoEEG/ECG monitoring over a 14 year period. Results Twentynine seizures with ictal bradycardia were identified in 13 patients. Of these, 11 seizures in seven patients were associated with asystole. Bilateral transient limb movements consisting of asymmetric posturing or jerking occurred during eight episodes of ictal asystole, and loss of muscle tone occurred during four. In contrast, neither bilateral limb movement nor loss of muscle tone was observed in any of the non-asystolic seizures, whether ictal bradycardia was present or not. All patients with ictal asystole reported a history of seizure-related falls and brief body and limb jerking. Conclusions The presence of loss of muscle tone or bilateral asymmetric jerky limb movements during a seizure suggests the possibility of ictal asystole. VideoEEG/ECG monitoring should be considered in patients with epilepsy demonstrating these clinical features to determine if ictal asystole is present.

Key words epilepsy . temporal lobe syncope . bradycardia heart arrest . autonomic nervous system diseases $\cdot$ autonomic nervous system

\section{Introduction}

Ictal asystole, the occurrence of asystole during a seizure, is the most dramatic manifestation of ictal bradycardia and may contribute to seizure-related injury and sudden unexplained death in epilepsy patients (SUDEP) [5]. Optimal control depends on recognition and treatment of both the epileptic and bradycardic components of the disorder. While for- merly thought to be rare [22], ictal bradycardia may be more common than previously appreciated. When epilepsy patients were monitored with implantable loop recorders, ictal bradycardia was found in seven out of 19 patients, of whom four underwent cardiac pacemaker implantation [19].

The identification of ictal asystole can be challenging, but has important therapeutic implications. Most patients are not suspected of having the disorder until a clinical event is captured during prolonged 
Fig. 1 Case selection process. PPM = permanent pacemaker

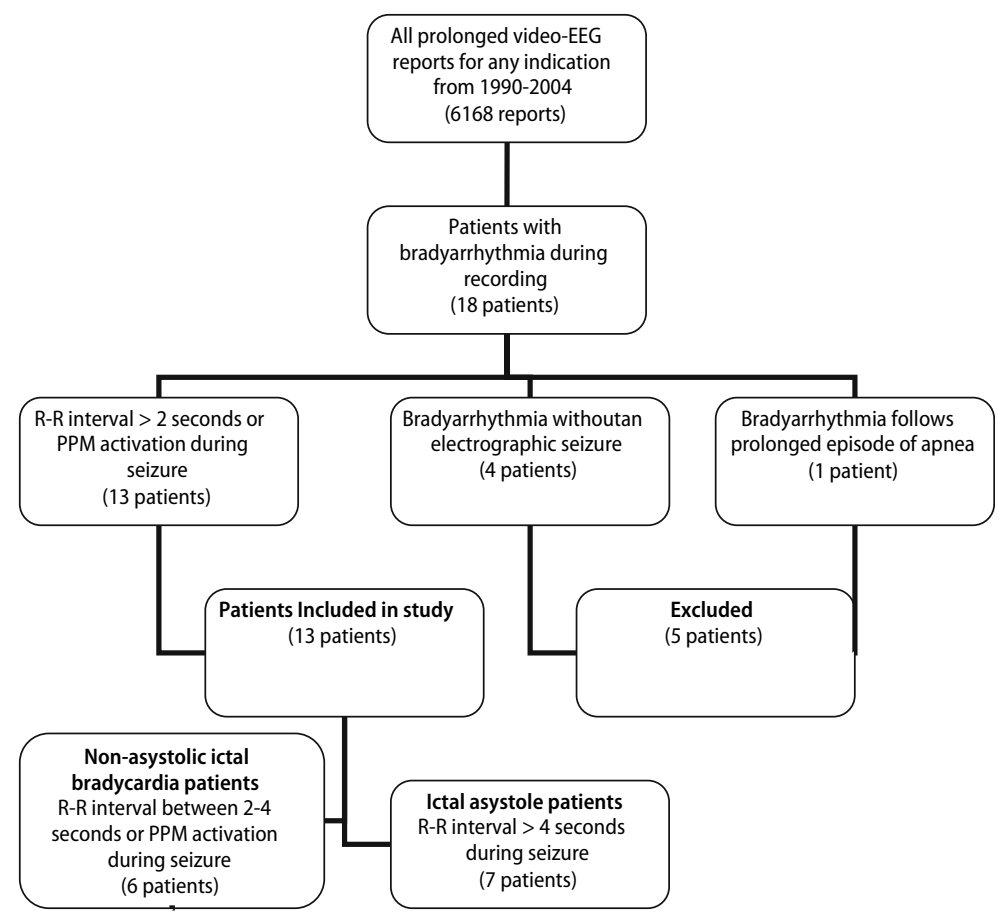

video EEG/ECG monitoring. Seizures and syncope are common, with a lifetime prevalence of $0.5 \%$ and $3 \%$ respectively [13, 20]. Performing prolonged video EEG/ECG monitoring in all epilepsy and syncope patients would be impractical. A focused analysis of the clinical features of ictal asystole in comparison to non-asystolic seizures has not been published and prior descriptions of observed episodes of ictal asystole are limited. The purpose of this study was to determine clinical cues to aid in the diagnosis of ictal asystole in order to identify patients who may benefit from evaluation with video-EEG/ECG monitoring.

\section{Patients and methods}

A database query was performed to identify ictal bradycardia events. All adult and pediatric EEG reports of prolonged video-EEG/ECG monitoring studies performed for any indication at Mayo Clinic Rochester between January 1990 and December 2004 were searched. Ictal bradycardia or asystole was defined as the occurrence of bradycardia or asystole during a recorded partial or generalized epileptic seizure, where the onset of the bradyarrhythmia began after the onset of the electrographic seizure discharge as seen on the prolonged video-EEG/ECG recording. Bradycardia was defined as an $\mathrm{R}-\mathrm{R}$ interval of greater than $2.0 \mathrm{~s}$ or activation of cardiac pacing for a decreased heart rate in patients previously implanted with a cardiac pacemaker. Asystole was defined as an $\mathrm{R}-\mathrm{R}$ interval of greater than $4.0 \mathrm{~s}$. Thirteen patients met the inclusion criteria for ictal bradycardia, of which seven had episodes that met the definition for ictal asystole. The case selection process is summarized in Figure 1. The pre-evaluation diagnoses and treatments are listed in Table 1. All patients authorized use of their medical information, and the study received approval of the Institutional Review Board.
All patients were evaluated with computer-assisted continuous 30 channel scalp EEG recordings using the International 10-20 system for electrode placement. One patient also underwent intracranial monitoring with an 8-contact depth electrode placed in each medial temporal region. A single channel was used to display the ECG in all cases.

All available videos and ECG/EEG findings of the recorded events in all cases were reviewed by two of the authors (G.G. and J.B) to identify semiologic findings and determine the duration of postictal confusion. Postictal reorientation time was defined as the time between the termination of the electrographic seizure activity and the time at which the patient was fully oriented or was able to correctly read from test cards [9]. Testing for reorientation time was considered sufficient if it was performed within $20 \mathrm{~s}$ of electrographic seizure offset and was continued until the patient performed the test correctly. The description of clinical features in the EEG reports had to be relied on for six of the 60 seizures, as the video data was no longer available. The ECG channel was obscured by artifact during two seizures. Sufficient testing of postictal reorientation was not performed during six of the complex partial seizures, and video data was not available for five. Comparisons of postictal reorientation times were made using the Wilcoxon ranksums test using JMP 6.0 software from SAS.

\section{Results}

The clinical features of the seizures associated with asystole were distinct from the non-asystolic complex partial seizures (see Table 1). All 11 recorded episodes of ictal asystole occurred during complex partial seizures. Ten of the $11 \mathrm{ictal}$ asystole seizures were associated with either brief jerky bilateral limb movements or a loss of muscle tone with collapse or head drop. The brief jerky bilateral limb movements 
Table 1 Clinical manifestations and pre-evaluation diagnosis

\begin{tabular}{|c|c|c|c|c|c|}
\hline ID & Pre-Eval. Dx & Pre-Eval. morbidities & Pre-Eval Tx & Asy dur & Description of seizures with ictal bradycardia \\
\hline \multicolumn{6}{|c|}{ Patients diagnosed with ictal asystole } \\
\hline A & Indeterminate syncope & Falls, 3 MVAs & None & 4 & stares; oral automatisms; head drop \\
\hline B & Epilepsy & Falls, fractured nose & CBZ+LEV & 10 & $\begin{array}{l}\text { out of body sensation; collapse backwards; brief bilateral } \\
\text { asymmetric upper extremity myoclonic jerks }\end{array}$ \\
\hline $\mathrm{C}$ & Epilepsy & Falls, jaw fracture & $\mathrm{LEV}+\mathrm{PB}+\mathrm{PHT}$ & 14 & behavioral arrest; bilateral limb posturing; head drop \\
\hline D & Epilepsy & Falls & LEV+PHT & 36 & behavioral arrest; oral automatisms; bilateral limb posturing \\
\hline $\mathrm{E}$ & Epilepsy & Falls with soft tissue injuries & $\mathrm{CBZ}+\mathrm{LEV}$ & 25 & behavioral arrest; bilateral limb posturing \\
\hline $\mathrm{F}$ & Epilepsy & Falls & LEV+PHT & 21 & aura; bilateral limb posturing; urinary incontinence \\
\hline G & Epilepsy & Falls & LTG+LEV & 29 & collapses backwards; urinary incontinence \\
\hline \multicolumn{6}{|c|}{ Patients diagnosed non-asystolic ictal bradycardia } \\
\hline $\mathrm{H}$ & Epilepsy & None & VPA & & behavioral arrest \\
\hline I & Vasovagal syncope; panic attacks & $\begin{array}{l}\text { Falls with soft } \\
\quad \text { tissue injuries, } 1 \text { MVA }\end{array}$ & PPM & & epigastric and déjà vu sensation; behavioral arrest \\
\hline J & Vasovagal syncope; panic attacks & Falls & PPM & & olfactory sensation; behavioral arrest \\
\hline K & Epilepsy & Falls, 1 MVA & PHT & & indescribable sensation; stares; aphasic \\
\hline L & Epilepsy & Falls, 1 MVA & PHT+TPM & & $\begin{array}{l}\text { oral automatisms; left head version; } \\
\text { left upper extremity posturing }\end{array}$ \\
\hline M & Epilepsy & Falls & $\mathrm{LEV}+\mathrm{PHT}$ & & $\begin{array}{l}\text { behavioral arrest; oral and hand automatisms; } \\
\text { blinking; verbalizations }\end{array}$ \\
\hline
\end{tabular}

Pre-Eval. Dx = patient's diagnosis prior to evaluation for ictal bradycardia; Pre-Eval. Morbidities = seizure-related morbidities prior to evaluation for ictal bradycardia; MVA = seizure-related motor vehicle accidents; Pre-Eval Tx = treatment patient was on at the time they presented for evaluation; $C B Z=$ carbamazepine; LEV = levetiracetam; PB = phenobarbital; PHT = phenytoin; LTG = lamotrigine; VPA = valproic acid; PPM = permanent cardiac pacemaker; TPM = topiramate; Asy Dur = asystole duration in seconds; In patients with multiple recorded episode of ictal bradycardia, the seizure with the longest duration of asystole or bradycardia was described in the table

occurred during eight episodes of ictal asystole in five patients. The bilateral limb movements were characterized by brief asymmetric tonic extensor or flexor posturing of both upper extremities. These were sometimes associated with extension of the trunk resembling opisthotonus, or irregular bilateral jerking movements similar to myoclonus. During four of 11 episodes of ictal asystole in four patients, we observed loss of muscle tone characterized by a head drop or collapse. During the other seven episodes of ictal asystole, it was not possible to determine whether there was a loss of muscle tone, as the patients were lying supine in bed. In one patient, no distinctive clinical features occurred during one $10 \mathrm{~s}$ episode of ictal asystole, but this same patient had an additional $21 \mathrm{~s}$ episode of asystole associated with bilateral limb posturing. Urinary incontinence occurred during two asystolic episodes in two different patients.

In contrast, none of the non-asystolic seizures correlated with bilateral limb movements, loss of muscle tone, or urinary incontinence. The clinical features of seizures without bradycardia and those with non-asystolic bradycardia were indistinguishable and showed clinical findings typical of complex partial seizures. All but one of the patients with asystolic seizures also demonstrated semiologic findings typical of complex partial seizures, but these features preceded the asystole and bilateral limb movements or loss of muscle tone.

The EEG pattern of ictal asystole revealed bilateral high amplitude slowing which was usually followed by attenuation. All of the ictal asystole patients had one or both of these EEG findings. In contrast the EEG recordings during seizures associated with nonasystolic bradycardia were indistinguishable from those seizures not associated with bradycardia and did not demonstrate the bilateral high amplitude slowing and attenuation seen during ictal asystole.

The postictal reorientation time could be calculated for 28 out of 39 recorded complex partial seizures, 6 with asystole and 22 without asystole. The mean reorientation time for complex partial seizures without asystole ( $82 \mathrm{~s}$, range $4-163 \mathrm{~s}$ ) was not statistically different from the mean reorientation time seen following ictal asystole seizures $(54 \mathrm{~s}$, range 5-60 s, $P=0.59$, Wilcoxon rank-sums test).

The features of the seizures described in clinical histories of the ictal asystole patients correlated with the semiologic findings observed during video-EEG/ ECG monitoring. All of the patients diagnosed with ictal asystole were reported to have witnessed episodes of loss of consciousness with seizure-related falls and episodes of brief jerking movements of the body and extremities in their history prior to admission to the video EEG/ECG monitoring unit. Two ictal asystole patients were reported to manifest pallor during their seizures, and the wife of one patient reported that the patient had a "flat line" on an emergency room ECG monitor during a clinical seizure. No patient had undergone previous attempts at cardiopulmonary resuscitation during a clinical event. 
Table 2 Radiology and seizure characteristics

\begin{tabular}{|c|c|c|c|c|c|c|c|c|c|c|}
\hline ID & Age/sex & Dur & Radiology & $\begin{array}{l}\text { Total } \\
\text { Sz }\end{array}$ & $\begin{array}{l}\text { No. of } \\
\text { CPS }\end{array}$ & $\begin{array}{l}\text { No. of } \\
\text { SPS }\end{array}$ & $\begin{array}{l}\text { No. of } \\
\text { GTC }\end{array}$ & $\begin{array}{l}\text { No. of } S z \\
\text { with } I B / I A\end{array}$ & $\begin{array}{l}\text { EEG at } \\
\text { Sz onset }\end{array}$ & $\begin{array}{l}\text { EEG at } \\
\text { IB onset }\end{array}$ \\
\hline \multicolumn{11}{|c|}{ Patients diagnosed with ictal asystole } \\
\hline A & $72 / \mathrm{F}$ & $3 y$ & Normal & 1 & 1 & 0 & 0 & $0 / 1$ & LT & BT \\
\hline B & $52 / \mathrm{F}$ & $14 \mathrm{y}$ & Left temporal glioma & 1 & 1 & 0 & 0 & $0 / 1$ & LT & LT \\
\hline $\mathrm{C}$ & $25 / M$ & $5 y$ & Bilateral mesial temporal sclerosis & 18 & 11 & 7 & 0 & $3 / 1$ & 3BT; 1 LT & BT \\
\hline D & 40/M & $8 y$ & Bilateral mesial temporal sclerosis & 4 & 4 & 0 & 0 & $0 / 4$ & 2 LT; 2 ID & $2 \mathrm{LT} ; 2 \mathrm{ID}$ \\
\hline $\mathrm{E}$ & $17 / M$ & $18 \mathrm{~m}$ & Normal & 2 & 1 & 1 & 0 & $0 / 1$ & RT & BT \\
\hline $\mathrm{F}$ & $29 / F$ & $21 y$ & Normal & 2 & 2 & 0 & 0 & $0 / 2$ & RT & BT \\
\hline G & $70 / F$ & 57 y & Nonenhacing $\mathrm{R}$ temporal mass & 4 & 4 & 0 & 0 & $0 / 1$ & RT & BT \\
\hline \multicolumn{11}{|c|}{ Patients diagnosed with non-aystolic ictal bradycardia } \\
\hline $\mathrm{H}$ & $67 / F$ & $9 \mathrm{~d}$ & $\begin{array}{l}\text { Subacute left subinsular infarct, } \\
\text { coiled ICA aneurysm }\end{array}$ & 1 & 1 & 0 & 0 & $1 / 0$ & LT & LT \\
\hline I & $35 / \mathrm{F}$ & $5 y$ & Normal (CT with contrast) & 8 & 3 & 5 & 0 & $8 / 0$ & RT & RT \\
\hline J & $54 / \mathrm{F}$ & $8 \mathrm{~m}$ & Normal & 1 & 1 & 0 & 0 & $1 / 0$ & RT & BT \\
\hline K & $73 / \mathrm{M}$ & $8 \mathrm{y}$ & Right caudate lacunar infarct & 3 & 1 & 2 & 0 & $3 / 0$ & RT & BT \\
\hline L & $28 / M$ & $12 y$ & Heterotropia of right occipital horn & 6 & 4 & 0 & 2 & $1 / 0$ & RT & BT \\
\hline \multirow[t]{2}{*}{ M } & $33 / F$ & 4 y & Normal & 9 & 5 & 4 & 0 & $1 / 0$ & LT & BT \\
\hline & Mean 45.7 & $10.5 \mathrm{y}$ & & Total 60 & 39 & 19 & 2 & 18/11 & & \\
\hline
\end{tabular}

Age = age at diagnosis of ictal bradycardia; Dur = duration of seizure disorder; $y=$ years; $m=$ months; $d=$ days; Radiology = MRI unless specified; ICA $=$ internal carotid artery; Total $\mathrm{Sz}=$ total number of seizures recorded; No. of CPS = number of complex partial seizures recorded; No. of SPS $=$ number of simple partial seizures recorded; No. of GTC = number of secondarily generalized tonic-clonic seizures recorded; No. of Sz with IB/IA = number seizures associated with non-asystolic ictal bradycardia (IB) and with ictal asystole (IA); EEG at Sz onset = localization of seizure activity at the onset of the seizures associated with ictal bradycardia; LT = left temporal; RT = right temporal; $\mathrm{BT}=$ bitemporal; ID = indeterminate; $\mathrm{EEG}$ at IB onset = localization of seizure activity at the onset of bradycardia

The patients with ictal asystole and non-asystolic bradycardia were similar in age, radiological characteristics, and lateralization of seizure onset (see Table 2). There was no difference in beta-blocker or antiepileptic drug use between these groups, except for increased levetiracetam use in the patients with ictal asystole (see Tables 1, 3).

\section{Discussion}

This review of clinical features reveals that attacks of ictal asystole were clinically associated with loss of muscle tone or brief arrhythmic bilateral upper extremity posturing and jerking that was distinct from the rhythmic and more sustained tonic-clonic convulsive activity and dystonic posturing typically seen in the context of seizures uncomplicated by cardiac arrhythmias. The historical data provided by our patients prior to prolonged video-EEG/ECG monitoring also suggested the presence of the motor activity associated with ictal asystole and in some cases also suggested a hemodynamic component to the witnessed seizures. Thus, this study suggests that there are clinical cues in patients with ictal asystole that can be obtained upon careful history taking which may allow identification of patients from the general epilepsy population who may benefit from evaluation with prolonged EEG/ECG monitoring. Although a prospective study design comparing ictal asystole and non-asystolic seizures would be ideal in determining the relative sensitivity and specificity of these signs, ictal asystole is uncommon enough that such a study would be difficult.

The brief bilateral upper extremity posturing and loss of tone observed in our patients are not specific for ictal asystole and may be seen in syncope from other causes. For example, one video study of healthy subjects during induced syncope showed a 90\% incidence of bilateral synchronous and asynchronous jerking and myoclonus during video-recorded attacks of syncope, similar in description to the observations in our patients [12]. Other studies of vasovagal syncope have described these movement as clonic jerks, tonic spasms, or decerebrate or decorticate posturing [1]. The similarity of the clinical features of nonepileptic syncope and ictal asystole suggests that the clinical manifestations of ictal asystole most likely are the result of cerebral hypoperfusion secondary to asystole rather than from seizure-induced stimulation of cortical and subcortical regions. In addition, the bilateral high amplitude slowing and suppression of the EEG which developed following asystole is typical of the EEG findings during non-epileptic syncope [1].

There were some technical limitations that affected our observations. As the majority of the recorded clinical events occurred with the patient supine in bed, our study likely underestimated the incidence of loss of postural tone during ictal asystole. In addition, many of the seizures were observed in black and white video and some using infrared light, limiting our ability to look for the presence or absence of pallor 
Table 3 Cardiac testing and medications in patients

\begin{tabular}{|c|c|c|c|c|c|}
\hline ID & 12 lead EKG & Echo & Holter & Head upright tilt & CV meds \\
\hline \multicolumn{6}{|c|}{ Patients diagnosed with ictal asystole } \\
\hline A & Normal & NA & Normal & NA & none \\
\hline B & Sinus $B C$ at 45 & Normal & Normal & NA & atenolol \\
\hline C & Normal & NA & NA & NA & none \\
\hline D & A fib & Normal & Parox a fib & NA & none \\
\hline $\mathrm{E}$ & Normal & Normal & Normal & NA & none \\
\hline $\mathrm{F}$ & Normal & NA & NA & Symptomatic hypotension & none \\
\hline G & Sinus $B C$ at 55 & Normal & NA & NA & propanolol \\
\hline \multicolumn{6}{|c|}{ Patients diagnosed with non-asystolic ictal bradycardia } \\
\hline H & RBBB & Normal & NA & NA & none \\
\hline I & Normal & Normal & Normal & Symptomatic hypotension & none \\
\hline J & Normal & NA & Normal & Symptomatic hypotension & metoprolol \\
\hline K & Sinus $B C$ at 55 & Normal & NA & NA & none \\
\hline L & NA & NA & NA & NA & metoprolol \\
\hline M & Normal & NA & Normal & NA & none \\
\hline
\end{tabular}

$\mathrm{BC}=$ bradycardia; $\mathrm{A}$ fib = atrial fibrillation; NA = not available; Echo = echocardiogram; Parox a fib = paroxysmal atrial fibrillation; CV Meds = cardiovascular medications the patient was on at the time of ictal bradycardia diagnosis

during the attacks of ictal syncope and non-asystolic seizures. One might predict that pallor would be more common in ictal syncope than in other seizures, but this could not be determined in our study. It has been suggested that prolonged post-ictal confusion might be a clue to the diagnosis of ictal bradycardia [17]. However, the post-ictal recovery period in ictal asystole and non-asystolic seizures was not significantly different in our series. The lack of a prolonged reorientation time for the patient with ictal asystole is similar to what has been observed in syncope from other causes [18].

The diagnosis of ictal asystole requires the recording of a representative clinical event during simultaneous video-EEG/ECG monitoring [2]. We observed that our patients and other reported ictal bradycardia cases do not have bradycardia with every seizure $[10,15,19,21]$. Therefore, a sufficient number of seizures need to be recorded to be reasonably certain that ictal asystole is not present. In a study where partial epilepsy patients were monitored with implantable loop recorders for several months, those diagnosed with ictal bradycardia only had bradycardia during $5-6 \%$ of the total recorded seizures [19]. Our study was not designed to determine the prevalence of ictal asystole and cannot establish the number of seizures which must be recorded to assure correct diagnosis. Further studies with long-loop ECG recordings in epilepsy patients may be necessary to answer this question.

Recognition of ictal asystole is important for those who evaluate patients with epilepsy, syncope, and other episodic disorders. Ten of the 13 patients identified in this series had a diagnosis of epilepsy without a suspicion of a bradyarrhythmia, and the diagnosis would have been missed without simultaneous video-EEG/ECG monitoring. In two of our cases and in six ictal bradycardia patients in the literature, ictal bradycardia was diagnosed only after cardiac pacing failed to control symptoms in patients previously diagnosed with cardiogenic or vasovagal syncope based on ECG monitoring alone [4, 6, 7, 11, 16]. Prolonged ECG monitoring without EEG may mislead the physician, as the finding of bradycardia coincident with a logged clinical event during prolonged ECG monitoring may suggest a primary cardiogenic diagnosis [14]. The clinical features of ictal bradycardia can also be confused with other episodic disorders such as panic attacks, as occurred in two of our patients. Panic attack presentation has been associated with right temporal seizures, and has been reported in association with ictal bradycardia [21]. While it is impractical to perform prolonged videoECG/EEG monitoring in all patients with seizures, syncope, or other episodic disorders, this should be considered where clinical events persist despite appropriate therapy. Ictal asystole patients who are thought to have treatment resistant syncope may require antiepileptic drug therapy, and intractable epilepsy patients may require treatment for a seizureinduced bradyarrhythmia. In our series all seven ictal asystole patients reported falls prior to diagnosis. After cardiac pacing, only one patient had a seizure related fall with a mean follow up duration of 27 months [8].

It is possible that patients in whom ictal asystole occurs may be inherently prone to vagally-mediated phenomena such as bradycardia, with seizures serving as a nonspecific provocative factor. While autonomic studies were essentially normal in one series of ictal bradycardia patients [22], three of our patients had transient abnormalities during tilt table testing which were considered consistent with a diagnosis of neurally mediated syncope. During implantable loop 
recording in epilepsy patients, one of the seven patients with ictal bradycardia also had an episode of bradycardia in the absence of an identified seizure [19]. Further evaluation of autonomic and cardiovagal function in these patients may be useful. In contrast to ictal asystole patients who may have a susceptibility to vasodepressor effects which is manifested during epileptic seizures, an even more rare condition has been described where epileptic seizures occur during reoxygenation following episodes of syncope. These patients are theorized to have a lower seizure threshold, and the period of hypoxia-ischemia provokes the seizure [1].

Ictal bradycardia occurs most often in the setting of temporal lobe epilepsy, and has only been described during partial seizures [3]. It has been hypothesized that seizures may lead to the stimulation of regions of the brain, such as the insula, cingulate cortex, amygdala, or hypothalamus which regulate cardiac outflow through connection to brainstem and spinal cord nuclei [2]. Some have suggested ictal bradycardia may have lateralizing value as well. However our analysis of the dynamic EEG/ECG recordings of patients with ictal bradycardia in a previous publication suggested that bitemporal activation was present at bradycardia onset in the majority of patients, providing no clear evidence for the existence of a consistently lateralized cardiac parasympathetic representation in the central autonomic network [3].

\section{References}

1. Brenner RP (1997) Electroencephalography in syncope. J Clin Neurophysiol 14:197-209

2. Britton JW, Benarroch E (2006) Seizures and syncope: anatomic basis and diagnostic considerations. Clin Auton Res 16:18-28

3. Britton JW, Ghearing GR, Benarroch E, Cascino GD (2006) The Ictal Bradycardia Syndrome: Localization and Lateralization. Epilepsia 47:1-8

4. Constantin L, Martins JB, Fincham RW Dagli RD (1990) Bradycardia and syncope as manifestations of partial epilepsy. J Am Coll Cardiol 15:900-905

5. Dasheiff RM, Dickinson LJ (1986) Sudden unexpected death of epileptic patient due to cardiac arrhythmia after seizure. Arch Neurol 43:194-196

6. Devinsky O, Price BH, Cohen SI (1986) Cardiac manifestations of complex partial seizures. Am J Med 80:195-202

7. Fincham RW, Shivapour ET, Leis AA, Martins JB (1992) Ictal bradycardia with syncope: a case report. Neurology 42:2222-2223

8. Ghearing GR, Munger TM, Jaffe AS, Benarroch EE, Britton JW (2006) Ictal bradycardia: seizure semiology and treatment with cardiac pacing. Ann Neurol 60(Suppl 10):S-111
9. Goldberg-Stern H, Gadoth N, Cahill W, Privitera M (2004) Language dysfunction after frontal lobe partial seizures. Neurology 62:1637-1638

10. Kahane P, Di Leo M, Hoffmann D, Munari C (1999) Ictal bradycardia in a patient with a hypothalamic hamartoma: a stereo-EEG study. Epilepsia 40:522-527

11. Kowalik A, Bauer J, Elger CE (1998) Asystolic seizures. Nervenarzt 69:151157

12. Lempert T, Bauer M, Schmidt D (1994) Syncope: a videometric analysis of 56 episodes of transient cerebral hypoxia. Ann Neurol 36:233-237

13. MacDonald BK, Cockerell OC, Sander JWAS, Shorvon SD (2000) The incidence and lifetime prevalence of neurological disorders in a prospective community-based study in the UK. Brain 123:665-676

14. Maramattom BV, Britton JW, Ghearing GR, Wijdicks EF (2004) Ictal bradycardia after endovascular treatment of an aneurysm. J Neurosurg 101:546

15. Pritchett EL, McNamara JO, Gallagher JJ (1980) Arrhythmogenic epilepsy: an hypothesis. Am Heart J 100:683-688
16. Reeves AL, Nollet KE, Klass DW, Sharbrough FW, So EL (1996) The ictal bradycardia syndrome. Epilepsia 37:983-987

17. Rocamora R, Kurthen M, Lickfett L, von Oertzen J, Elger CE (2003) Cardiac asystole in epilepsy: clinical and neurophysiologic features. Epilepsia 44:179-185

18. Rossen R (1943) Acute arrest of cerebral circulation in man. Arch Neurol Psychiatry 50:510-528

19. Rugg-Gunn FJ, Simister RJ, Squirrell M, Holdright DR, Duncan PJS (2004) Cardiac arrhythmias in focal epilepsy: a prospective long-term study. Lancet 364:2212-2219

20. Savage DD, Corwin L, McGee DL (1985) Epidemiologic features of isolated syncope: the Framingham Study. Stroke 16:626-629

21. Sazgar M, Carlen PL, Wennberg R (2003) Panic attack semiology in right temporal lobe epilepsy. Epileptic Disord 5:93-100

22. Tinuper P, Bisulli F, Cerullo A, Carcangiu R, Marini C, Pierangeli G, Cortelli P (2001) Ictal bradycardia in partial epileptic seizures: autonomic investigation in three cases and literature review. Brain 124:2361-2371 